\title{
Current and Future Applications of Distributed Acoustic Sensing as a New Reservoir Geophysics Tool
}

\author{
Meng $\mathrm{Li}^{1}$, Hua Wang ${ }^{1,3, *}$ and Guo Tao ${ }^{1,2}$
}

${ }^{I}$ State Key Laboratory of Petroleum Resources and Prospecting, China University of Petroleum, Beijing, 102249,
China; ${ }^{2}$ Department of Petroleum Geosciences, The Petroleum Institute, P.O. Box 2533, Abu Dhabi, UAE; ${ }^{3}$ Earth Re-
sources Laboratory, Massachusetts Institute of Technology, Cambridge, MA, 02139, United States

\begin{abstract}
Distributed Acoustic Sensing (DAS) is a novel technique with low cost, no production deferment, complete coverage and repeatability for seismic data acquisition in vertical seismic profile (VSP), hydraulic fracturing monitoring, well and reservoir surveillance and micro-seismic detection. In this paper, we give a review on the field applications of DAS and the corresponding pre-processing methods as well as the limitations that hinder its further applications in exploration and production. Finally, future developments for DAS are discussed, including the enhancement of $\mathrm{S} / \mathrm{N}$ ratio, precise determination of receiver channels in depth, rapid processing of massive data and integrated interpretation of multi-mode optical fiber.
\end{abstract}

Keywords: DAS, hydraulic fracturing monitoring, microseismic, pre-processing methods, VSP.

\section{INTRODUCTION}

Distributed Acoustic Sensing (DAS) is a novel technique deploying an optical fiber cable instead of geophones to record the acoustics. The measurements are obtained by interrogating the back scattered signals which are associated with the strain along the fiber caused by the propagation of elastic waves. Once the spacing between adjacent channels in the fiber is determined, the Distributed Acoustic Sensing can be viewed as an array of dense 1-component receivers to measure the deformation along the fiber.

The first DAS operation was performed by Shell in 2009 and its capacity of acquiring the VSP measurements was reported by Mestayer et al. [1]. Since then, this technique has been improved and widely tested in various field situations including the VSP [2-7], microseismic measurements [8,9], well and reservoir surveillance [10], hydraulic fracturing monitoring and diagnostics [11-13].

During the applications, several advantages of DAS over geophones have been verified. First of all, DAS is not limited in a horizontal well or ultra-slim well due to its slim cable. In addition, the cost of optical fiber is relatively low compared to geophones and the fiber cable is much easier to be installed together with other optical fiber sensors like Distributed Temperature Sensing (DTS) and Distributed Pressure Sensing (DPS). Moreover, unlike a geophone, DAS is able to obtain the measurements with complete vertical coverage of a well without movements of the fiber,

\footnotetext{
*Address correspondence to this author at the No. 18, FuXue Road, Beijing, China. Postcard: 102249; Tel: +86 89733754;

E-mail: wanghuaupc@126.com
}

therefore, the data continuity is ensured. Finally, DAS is non-intrusive, which means it can be used in exploration well, production well and observation well without production deferment. Although DAS has significant advantages, some limitations including the low S/N ratio, uncertainty in channel depth, lack of transverse sensitivity and only one-component measurements still limit its further applications.

In this paper we review the recent applications and some unique pre-processing techniques of DAS. First, we briefly explain how DAS works and show some field data examples particularly on VSP, microseismic measurements, hydraulic fracturing monitoring and diagnostics. Then, the limitations of DAS and some corresponding pre-processing techniques are reviewed. Finally, we conclude with a discussion on DAS challenges and possible solutions.

\section{WORKING PRINCIPLES OF DISTRIBUTED ACOUSTIC SENSING MEASUREMENTS}

\subsection{Categories of Optical Fiber Technique}

Optical fiber sensing technologies were first introduced in the 1990s for measurements of pressure and temperature using single point sensors. With further development, three types of optical fiber have been extensively applied in Oil \& Gas industry. Fig. (1) shows the types of optical fiber techniques. The first one is the Point Sensor (PS), where a single point sensor is installed at the end of the fiber for the measurements of pressure and temperature. The second one is Quasi-Distributed Sensor (QDS), where multiple point sensors are located at the fiber with certain spacing for the measurements of strain and temperature. The last one is the fully Distributed Sensor (DS), where the en- 
tire fiber becomes the sensors measuring and transmitting the parameters simultaneously. It means that a single optical fiber can replace hundreds or thousands of traditional single-point sensors. This type of optical fiber can be used to measure various physical quantities including pressure, temperature, strain and acoustics.

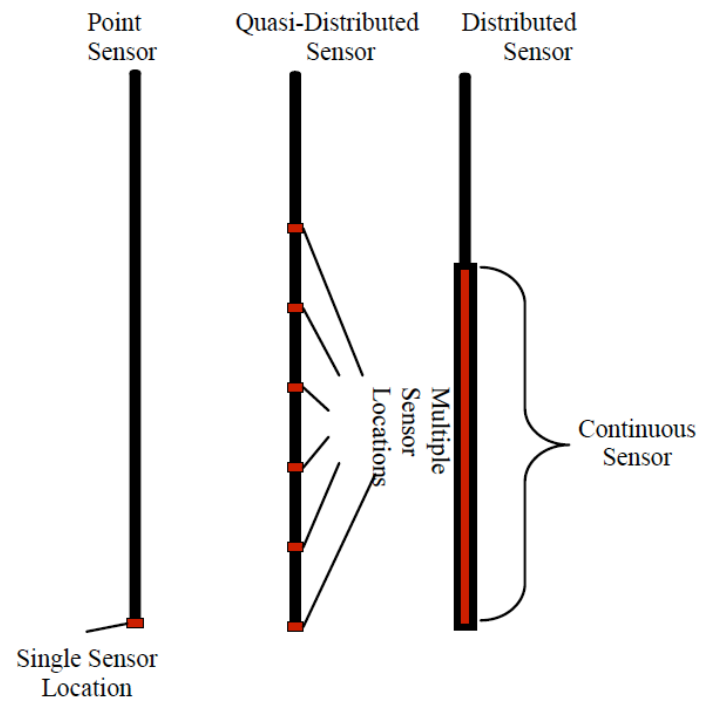

Fig. (1). Categories of optical fiber technique.

Among three types of optical fiber, Distributed Sensing is of great significance in geophysical measurements in downhole applications like hydraulic fracturing monitoring, VSP and microsiesmic measurements due to its entirely vertical converage of the wellbore. Distributed Temperature Sensing (DTS) and Distributed Acoustic Sensing (DAS) are two major applications of DS. The former technique uses Raman backscatter measuring the temperature and the latter one uses Rayleigh backscatter measuring the acoustics [13].

\subsection{Working Principles of DAS Measurements}

The typical DAS system is composed of an Interrogator Unit (IU) located at the surface linked with a standard optical fiber installed in a well or subsurface at a certain depth. The Interrogator Unit emits the short laser pulse travelling along the fiber and receives the back-scattered signals. By analyzing the phase lag of the sum of two back-scattered signals between two locations nearby a DAS channel for two emissions of light pulses, we can tell where and how much the fiber is deformed. The phase lag is proportional to the strain. The deformation is usually caused by the propagation of seismic waves. The distance over the two locations nearing a DAS channel is called 'gauge-length', which is usually determined prior to the acquisition. The phase-lag is interrogated everywhere along the fiber, so there are no discrete sensors. Instead, the whole fiber becomes the sensors. Fig. (2) shows the working principle of a typical DAS system. For the first emission of light pulse, the back-scatted signal at the front and end of gauge-length is S1 (blue) and S2 (blue), respectively. For the second emission of light pulse (the fiber is already strained), the back-scatted signal at the front and end of gauge-length is S1' (red) and S2' (red), respectively. The phase-lag between $\mathrm{S} 1+\mathrm{S} 2$ and $\mathrm{S} 1{ }^{\prime}+\mathrm{S} 2$ ' corresponds to the strain along the fiber.

Compared to single point sensor, the data quality of DAS do not rely on the performance of single point sensor, and hence is not limited by a need for multiple fibers or optical multiplexing to avoid optical crosstalk between interferometers [12]. The generation rate of light pulse is much higher than seismic frequencies, typically in the range of $10-100 \mathrm{kHz}$. The rate can be adjusted and a higher rate corresponds to a high $\mathrm{S} / \mathrm{N}$ ratio, vice-versa. However, the maximum of the generating rate is limited by the length of fiber. Although DAS is continuously interrogated along the fiber, we can view DAS as an array of receivers with high density. The spacing between adjacent receivers cannot be

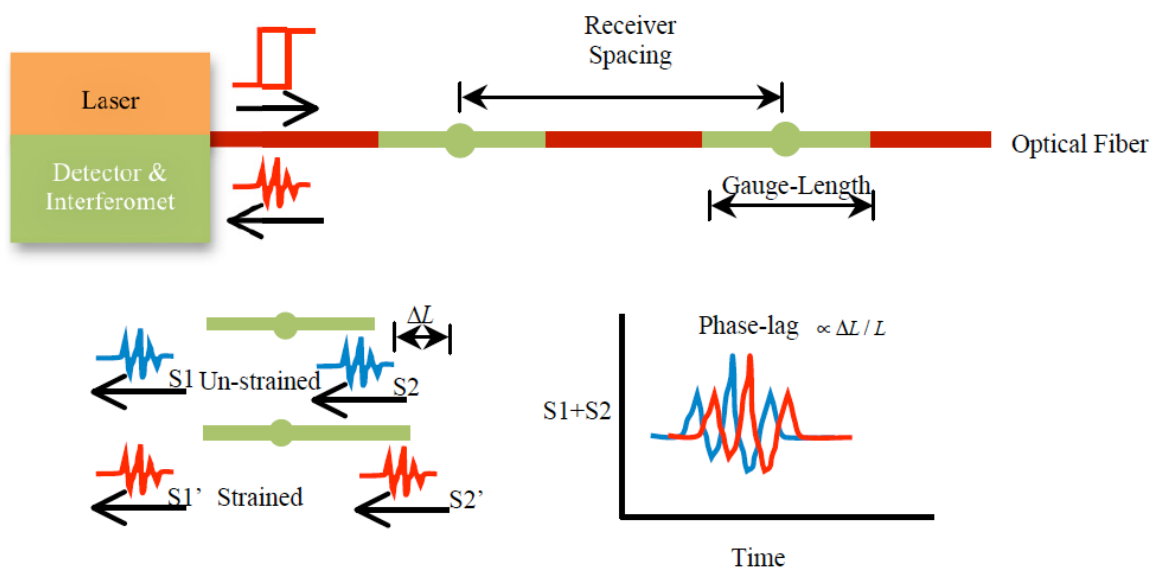

Fig. (2). Description and the explanation of working principle for DAS system [14]. "S1+S2" means the sum of the back-scattered signals at the front and end of gauge-length (un-strained) for the first emission of light pulse. "S1'+S2'" means the sum of the back-scattered signals at the front and end of gauge-length (strained) for the second emission of light pulse. By analyze the phase-lag between "S1+S2"and "S1'+S2", we can obtain the strain along the fiber. 
shorter than $1 \mathrm{~m}$ for the current technique. As an example, a $5 \mathrm{~km}$ DAS system can be interrogated with the rate of $20 \mathrm{kHz}$ every $5 \mathrm{~m}$ providing 1,000 independent recordings. Moreover, these parameters can be adjusted for optimized performance.

\subsection{Fiber Deployment}

The optical fiber used in DAS system can be deployed in multiple ways:

On casing: clamped to outside of the casing. This type of fiber installation can be used as permanent monitoring in downhole or subsea applications with highest cost and data quality.

On tubing: clamped to tubing. This kind of deployment can be viewed as the semi-permanent measurements with lower cost and data quality.

Inside tubing: deployed inside the tubing, which is retrievable with the lowest cost but provide with a much noisier data than the former two types of deployment.

Fig. (3) compares the cost, data quality and source effort of these three different types of deployment. The "source effort" here means the source energy required for the receivers to detect meaningful signals. Although a recent trial in Louisiana (US) suggests that the fiber installed in fluid-fill tubing would also be able to detect meaningful seismic signals [3], the data acquired from the fiber installed inside the tubing has the lowest $\mathrm{S} / \mathrm{N}$ ratio hence it needs more source effort and additional processing. Therefore, the type of fiber deployment should be chosen based on the specifics of each case, and should strike a balance between cost and data quality.

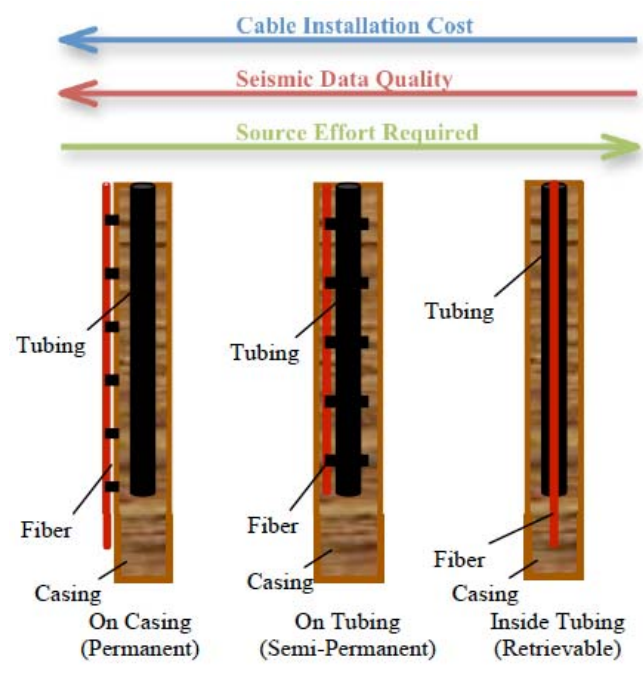

Fig. (3). Comparison of cost, data quality and source effort of three different types of fiber deployment.

\section{APPLICATIONS OF DISTRIBUTED ACOUS- TIC SENSING WITH GEOPHYSICAL MEAS- UREMENTS}

With the promising prospect and distinctive advantages, DAS has been tested widely over the life of the well includ- ing construction, completion and production. Applications of DAS in vertical seismic profile (VSP), microseismic measurements, well and reservoir surveillance, hydraulic fracturing monitoring and diagnostics demonstrate its capability as a replacement for geophones. Fig. (4) shows multiple applications of DAS during different stages over the life of the well.

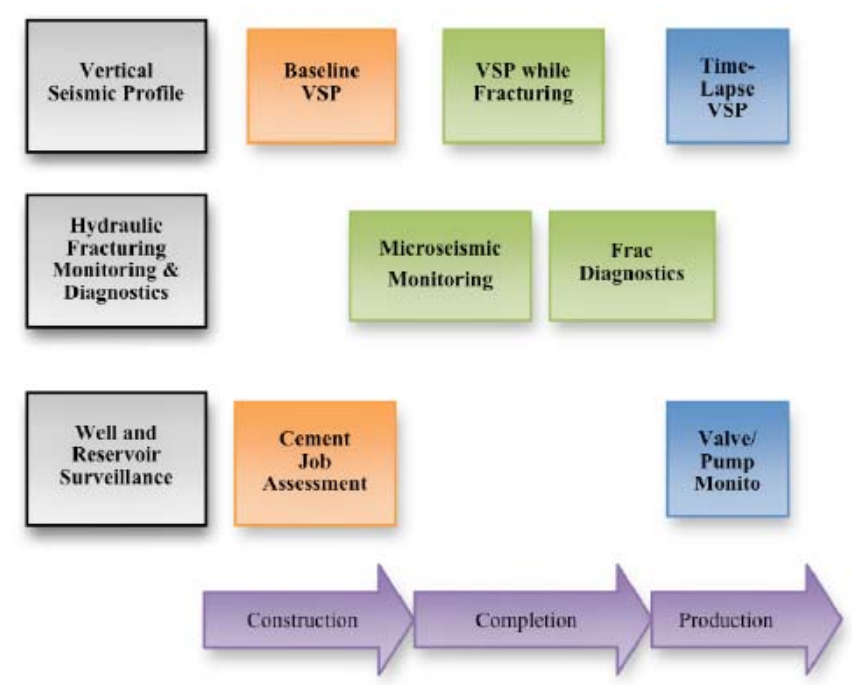

Fig. (4). Multiple applications for DAS.

\subsection{VSP}

The first DAS VSP field experiment was performed by shell in Canada in 2009. Subsequently, Mestayer et al. (2011) demonstrates its ability to replace geophones. Since then, 3D multi-well VSP, deep water time-lapse VSP and low-footprint monitoring have been tested with the DAS system developed by OptaSense [3, 4, 15], Silixa [2, 16-19], Schlumberger [20] and Halliburton [21].

Fig. (5) shows the comparison of zero-offset VSP data acquired from DAS and geophone arrays. The first arrivals from two separate acquirement systems are similar and strong. Overall, the DAS VSP is nosier than geophone VSP. The velocity obtained from DAS VSP, geophone VSP and sonic log are also compared. The DAS velocity matches well with the other two velocity profiles demonstrating the reliability of DAS VSP measurements. Fig. (6) shows a comparison of the VSP image obtained from DAS with geophone array. It's clear that the two images are very similar. The image of DAS VSP is narrower than that of geophone VSP. Here, "narrow" means that the length of interface in the image of DAS (red arrow) is shorter than that of geophone (blue arrow). Low $\mathrm{S} / \mathrm{N}$ ratio and poor transverse sensibility may be the reasons.

The most attractive advantage of DAS VSP is the low cost of optical fiber. Once the fiber is deployed, it serves as a permanent data acquirement system which is much cheaper than a geophone, and the survey is quicker with more extensive coverage. The avoidance of well intervention is another advantage for DAS VSP, making the VSP acquirement in treatment well without production deferment. 


\subsection{Microseismic Monitoring}

The first application of DAS for detecting micro-seismic events was performed by Shell [8-9]. In the field trail, they deployed the array of geophone and DAS in the same well aiming at comparing the ability and sensitivity of detecting the micro-seismic events during a stage of stimulation. Although the coverage of geophones is limited compared to DAS, their measurements recorded the same micro-seismic events with the similar arrival time as shown in Fig. (7). Due to the poor transverse sensibility, the $\mathrm{P}$ wave disappeared at the apex of the hyperbola on DAS records. However, with the full coverage of the entire well, DAS gives a wider aperture than geophone.

(a)

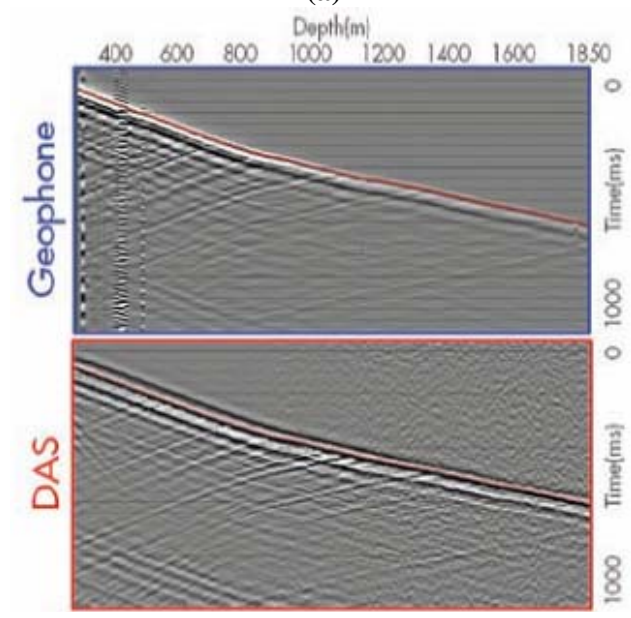

(b)

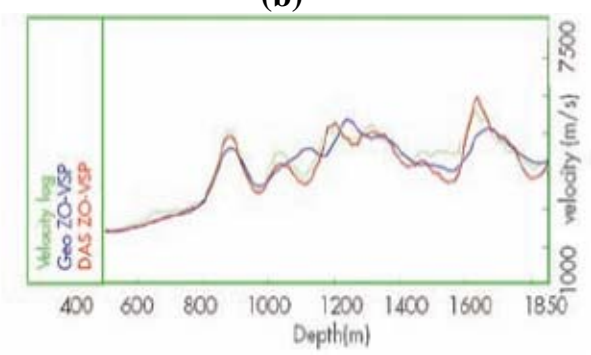

Fig. (5). (a) Zero-offset VSP recorded by a geophone array. Middle: Zero-offset VSP recorded by DAS array. (b) Comparison of velocity profiles obtained from geophone VSP (blue), DAS VSP (red) and sonic log (green) [1].

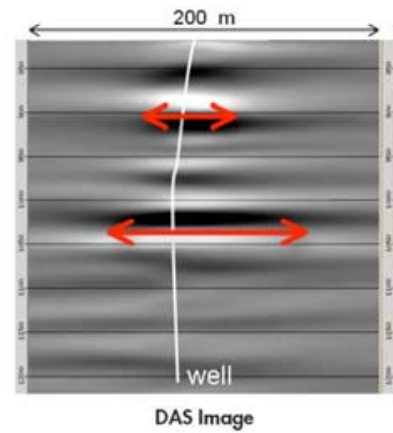

(a)

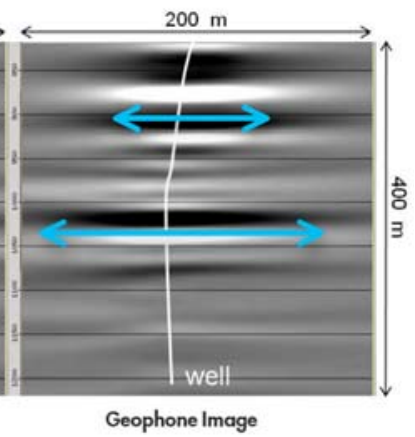

(b)
Fig. (6). (a) DAS VSP image of target area. (b) Geophone VSP image of target area [7].

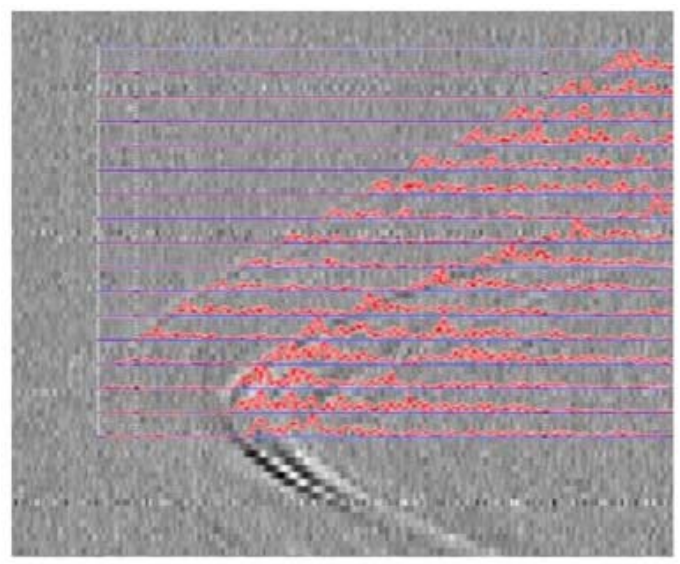

Fig. (7). A micro-seismic event detected on both DAS (grey) and geophone (red) data [8].

The main advantage of DAS micro-seismic application is that we can deploy the optical fiber in the treatment well, opening the potential of image of the micro-seismic activity in those harsh conditions where micro-seismic recordings are not possible in the observation well. In addition, it may give us a more precise micro-seismic event location using data both from treatment well and observation well. Finally, the location results combined with other information such as tracer data, pressure data and pump rates may be helpful for acquiring an integrated picture of hydraulic fractures.

Despite the advantages, the single component microseismic data obtained from DAS will be problematic in vertical wells because we can only determine the distance the event occurred from the wellbore without the knowledge of azimuth. The possible origin of the source locations formulates a circle. However, when the well is deviated or horizontal, we can narrow the possible source location to two points by drawing another circle, if the same micro-seismic data could be recorded in different DAS channels, as shown in Fig. (8).

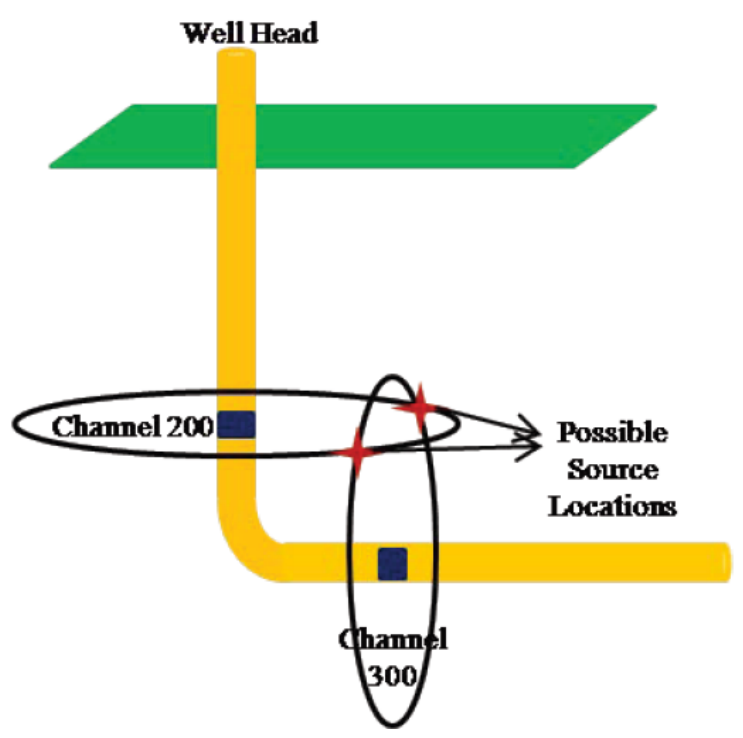

Fig. (8). Schematic diagram for narrowing the possible microseismic source locations in horizontal well [8]. 


\subsection{Hydraulic Fracturing Monitoring and Diagnostics}

Traditional hydraulic fracturing monitoring and diagnostics cover surface wellhead rates and pressures, downhole pressures and radioactive tracers [11]. These data are hard to obtain in complex reservoirs associated with shallow depth of investigation. To solve the problem, Distributed Temperature Sensing (DTS) is developed to give the engineers the real-time information [13]. Although the qualitative assessment of the placement of the fluid and proppant can be obtained from the DTS data during the stimulation, a quantitative estimation of injection rates and volumes is difficult to obtain [12]. To increase the confidence in interpretation of the hydraulic fracturing treatments, Distributed Acoustic Sensing (DAS) is applied. Combined with DTS, DAS and other information (pressures, rate and concentrations), we can have real-time insights on fracture initiation and growth, identification of perforation breakdowns, stage bypass events, fracture effectiveness and hydraulic fracture modelling. The field tests of DAS for hydraulic fracturing monitoring and diagnostics have been performed both in openhole multi-stage stimulation and cased \& cemented completion by Molenaar et al. 2011-2013. These tests showed that the combination of DTS and DAS can improve real-time hydraulic monitoring and stimulation diagnostics results.

Fig. (9) shows the distribution of DAS energy in interval 3 and 2 throughout the duration of the Stage 3 stimulation, which is performed at interval 3. The "intervals" here mean the isolated regions for stimulation in multi-stage hydraulic fracture process. Both of the intervals are isolated to prevent the liquid leaking. However, it's obvious that interval 2 seemed to be re-stimulated, which means that some fluid actually entered interval 2 during the stimulation of stage 3 . In addition, not all the fluid entered interval 3, which is the target zone. By estimating the volume of induced DAS energy (red color) in a certain interval, we can assess the volume of entered fluid. These DAS measurements provide the information of interval isolation and how it influences the ability to achieve the effective stimulation coverage.

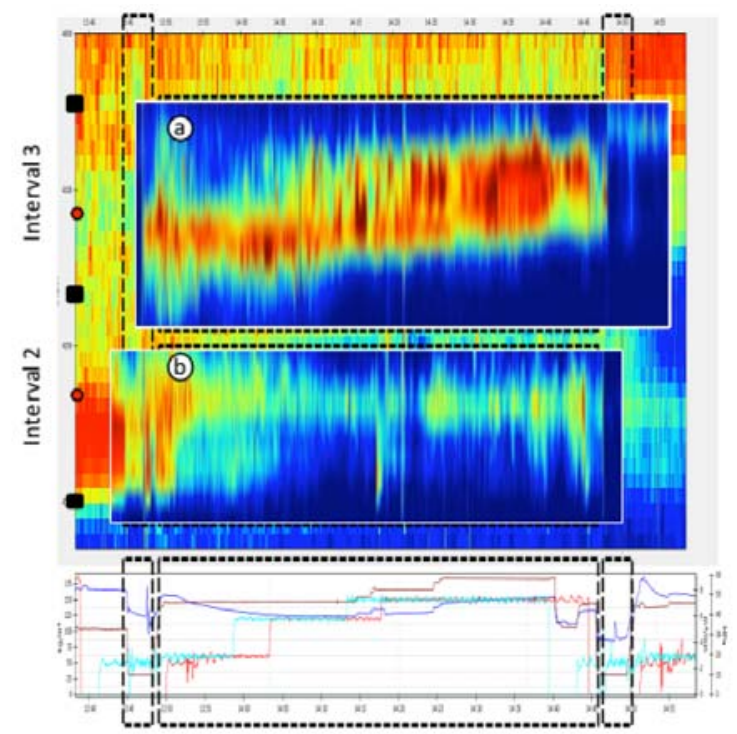

Fig. (9). DAS energy map showing the acoustic measurements for interval 2 and 3 during the stimulation of Stage 3 [12].
The benefit of hydraulic fracturing monitoring and diagnostics using DAS is the quantitative assessment of injection rates and the volume of injection fluid. This type of information is essential for optimizing the design of injection volume and improvement of hydraulic fracturing modelling. In addition, the introduction of DAS \& DTS aims to supplement reservoir monitoring data of high quality for the traditional fracturing monitoring and diagnostics techniques, making the real-time data transmission possible. Moreover, due to the limited coverage of traditional logging tools, the measurements for next stimulation stage need to move the acquisition system, therefore, the data are often delayed for several hours making it difficult to make correct decisions for engineers during the stimulation.

\subsection{Well and Reservoir Surveillance}

Besides the above applications, DAS can also replace the production logging tool to measure downhole production or injection flow performance in well and reservoir surveillance. The well surveillance data is not acquired in practice from traditional production logging tool due to the high cost with large operational risks and may cause the production deferment especially in a highly deviated or horizontal production well. In addition, in some cases, the small diameter production tubing limits the access of the production well logging tool. DAS applications in well and reservoir surveillance includes the estimation of production profile, injection profile, multiphase flow and well-integrity and production monitoring. Fig. (10) shows the production profiles estimated from DAS (blue) and production logging tool (red) for three different production periods in an unconventional gas producer. In Trial I and II, the DAS and production logging tool (PLT) measurements were done together before the installation of production tubing. In Trial III, the production tubing was already installed so the PLT was unable to access the inside of the borehole because of the small diameter of the tubing. Therefore, we could only obtain the DAS measurements. The axial flow estimated for the first two trials strongly indicate that the DAS can replace the PLT.
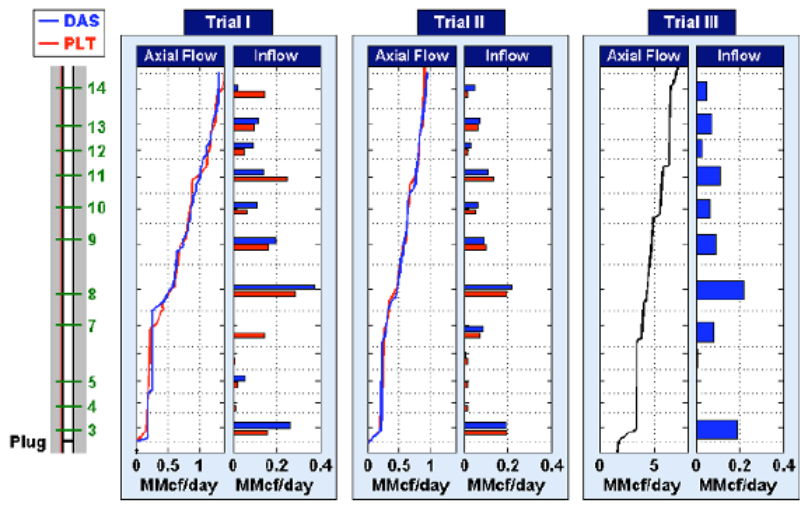

Fig. (10). Comparisons of production profile estimated from DAS (blue) and PLT (red) from three different production periods in an unconventional gas producer [10].

Well and reservoir surveillance using DAS can be viewed as permanent production logging tools once installed with various measurements using the same cable simply by 
applying different interrogator units and processing algorithms. In addition, DAS can provide with real-time measurements and visualization which will make a great contribution in optimization of stimulation.

\section{CHALLENGES AND LIMITATIONS OF DIS- TRIBUTED ACOUSTIC SENSING}

Although DAS has significant advantages over geophones, challenges and weaknesses still limit its applications.

\subsection{Low $S / N$ Ratio}

As mentioned before, the data acquired from DAS is much noisier than that of geophones. DAS noise is composed of random noise generated by the Interrogator Unit, the time-variant optical noise that occurs equally on all receivers and the spike-like noise [14]. The noise makes it difficult to detect weak signals especially on microseismic applications. Currently, we can reduce the impact of noise by simply stacking the data obtained from multiple optical fiber cables, if available, or apply some noise removal techniques [14]. But we still expect the enhancement of the noise floor of IU in the future. Fig. (11) shows the DAS and geophone datasets and compares the $\mathrm{S} / \mathrm{N}$ ratio for different windows: 1) shallow signal, 2) shallow noise, 3) deep signal and 4) deep noise. It's clear that the noise floor of DAS is higher than that of geophones.

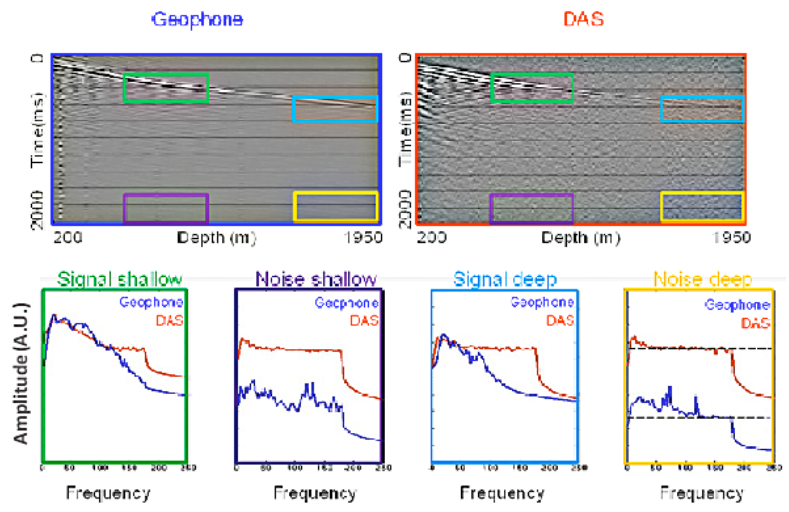

Fig. (11). Geophone (top left) and DAS (top right) data and comparison of scaled frequency spectra for signals at shallow depths (bottom left with green rectangle in geophone and DAS profile), noises at shallow depths (bottom middle-left with purple rectangle in geophone and DAS profile), signals at deep depths (bottom middle-right with blue rectangle in geophone and DAS profile), noise at deep depths (bottom right with yellow rectangle in geophone and DAS profile) [1].

\subsection{Depth Uncertainty}

The position of DAS channels in depth is determined by the arrival time of back-scattered light. However, the length of the fiber may be altered in the downhole application for some reasons. For example, the fiber may be bended due to its gravity in a horizontal well making the fiber itself stretched; the fiber may be longer than the measured depth to prevent snapping when descending along the well with the cable, etc. All these factors will lead to position uncertainty of DAS channels, causing the erroneous interpretation of seismic data. This limitation becomes an important issue in time-lapse DAS VSP applications. Depths of receivers should be accurate to compare the Normalized Difference in Root Mean Square (NDRMS) for observations of different time.

\subsection{Poor Transverse Fiber Sensibility}

DAS can only measure the strain along the fiber while the strain perpendicular to the cable can hardly be recorded, which means that DAS measurements are more sensitive to $P$ waves than $S$ waves when their propagation direction is parallel to the axis of fiber cable. As a result, information on $S$ waves may be absent in the near-offset DAS VSP and DAS microseismicity, where the shear wave is dominant and associated with induced fractures during the hydraulic fracturing [7]. Actually, the 1-C geophone is not immune to the problem of transverse sensibility. But the drop of DAS transverse sensibility is larger than that of geophone, as shown in Fig. (12), the transverse sensibility for DAS is approximate to $(\cos \theta)^{2}$ while that of geophone is close to $\cos \theta$ [13]. When the incident angle $(\theta)$ is zero, the wave travels along the inline axis and the amplitude of the $\mathrm{P}$ wave reaches its maximum. When the incident angle $(\theta)$ deviates zero, the amplitude of the $\mathrm{P}$ wave in DAS decreases faster than that of geophone.

This limitation seems to be serious in DAS VSP and DAS microseismicity. In near-offset VSP applications, information on the shear wave may be absent because its propagation direction is perpendicular to the fiber, while the DAS can only measure the strain along the fiber. In most cases, the shear wave has a close relationship with induced fractures, missing the shear wave may result in misjudgments during hydraulic fracturing [7]. In microseismic applications, when the source is located near the borehole, the energy of $S$ wave whose propagation direction is perpendicular to the axis of borehole will be decreased in DAS measurements. At the same time, when the source is located far from the borehole, $\mathrm{P}$ wave energy will be decreased too. This influence may make it difficult to pick up the effective event from the DAS micro-seismic data, resulting in the absence of some real source locations.

\subsection{Cable Deployment in Harsh Environments}

Although the deployment of optical fiber is neither complex nor expensive, the Interrogator Unit is hard to be permanently installed in harsh environment like the desert, the jungle, or subsea. The quality of recorded signals from permanent installed Interrogator Unit under such conditions is often degraded by the influence of sunlight exposure, storm or ocean currents. Therefore, IU must be made of high quality and robustness to survive these types of environments. In addition, the cost of installation of DAS should be further lowered as the technology is applied more extensively.

\section{DATA PROCESSING METHODS TO ALLE- VIATE THE LIMITATIONS OF DISTRIBUTED ACOUSTIC SENSING}

\subsection{Noise Removal}

Current de-noising technique includes the band-pass filter, median filter and stacking of multiple fiber 

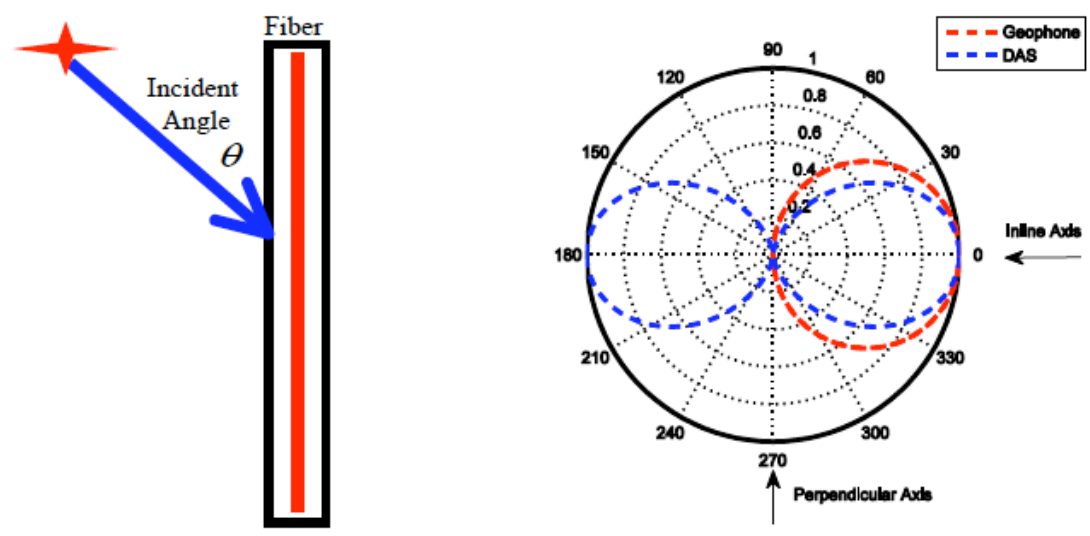

Fig. (12). Approximate amplitude-incident angle plots of DAS (blue) and geophone (red). The dashed line inside the circle refers to the normalized amplitude of the recorded $\mathrm{P}$ wave and the number around the circle refers to the incident angle. When the angle reaches $0^{\circ}$, the wave travels along the inline axis and the amplitude of the $\mathrm{P}$ wave reaches its maximum. When the incident angle deviates zero, for example, incident angle equals $30 \mathrm{o}$, the amplitude of the P wave in DAS is smaller than that of geophone.

measurements, which aims at removing the timevariant noise generated from temperature fluctuations in the well and optical noise $[14,22]$. Besides these background noises, large spike-like noise, frequently occurred at DAS VSP measurements, appears to be the dominant noise in raw DAS data [14]. Fig. (13) shows a field experiment utilizing DAS to record VSP measurements with Vibroseis source during stimulation in hydraulic fracturing. It's obvious that horizontal stripelike noise appears on both raw data (a) and correlated data (b). In addition, vertical stripe-like noise appears on correlated data especially above the plug (located at receiver\# 432) of the stimulation stage. These vertical stripe-like noises correspond to the spike-like noises in the raw data and appear in the entire time domain after correlation with Vibroseis source signal. The amplitude of the spike noise is much bigger than the background signal, making it possible to remove it in each trace by applying an amplitude threshold. For the waveform of each trace, every sample point whose amplitude is larger than the amplitude threshold is set to be zero. The threshold is calculated by the product of the median of the absolute amplitude values of all the sample points in the trace and a scale factor, as shown in equation 1. Here, Threshold,$\quad \alpha_{i}$ and $A m p_{i}^{j}$ refers to the amplitude threshold, scale factor and the amplitude for the $i$ th trace, respectively. $T$ is the total time sample number; $N$ is the total number of traces. This threshold processing technique is performed in each trace. Then, a median filter is employed to further suppress the remnant spikes and improve the S/N ratio. Fig. (14) compares the stacked correlated signal pre-stimulation (a) and post-stimulation (b) before noise removal and stacked correlated signal pre-stimulation (c) and poststimulation (d) after noise removal. It's clear that the vertical stripe-like noise has been successfully removed and reflections are recognizable.

Threshold $_{i}=\alpha_{i} \cdot \frac{\sum_{j=1}^{T}\left|A m p_{i}^{j}\right|}{T}, i=1: N$

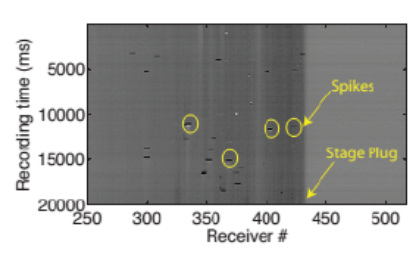

(a)

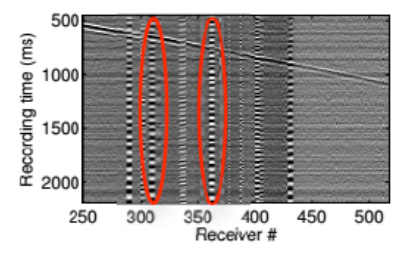

(b)
Fig. (13). (a) Raw DAS VSP data (b) Correlated Data in a certain hydraulic fracturing stage with the Vibroseis sweep signal [14]. The noises in red eclipse (vertical stripe-like noise) in correlated data (b) refer to the spikes in the raw DAS VSP data. These vertical stripe-like noises correspond to the spike-like noises in the raw data and appear in the entire time domain after correlation with Vibroseis source signal.

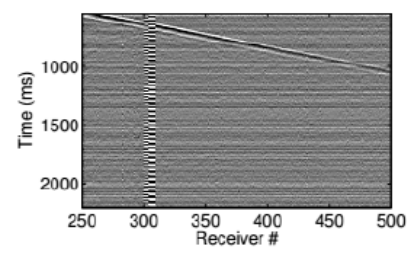

(a)

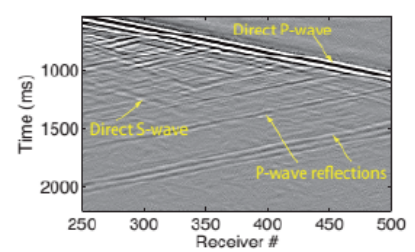

(c)

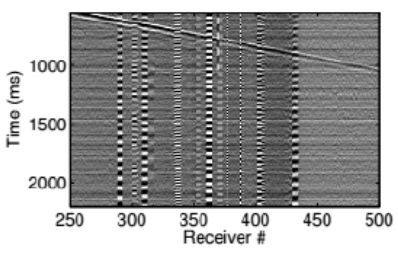

(b)

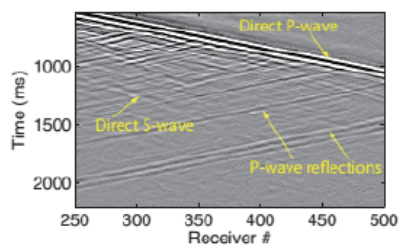

(d)
Fig. (14). stacked correlated signal pre-stimulation (a) and post-stimulation (b) before noise removal and stacked correlated signal pre-stimulation (c) and post-stimulation (d) after noise removal [14].

\subsection{Depth Calibration}

Depth calibration is another important issue for DAS measurements, especially in time-lapse applica- 
tions. Tube wave reflections at the plug and DC (Direct Current) level (mean of amplitude value of each trace) jump at the base of perforations are two separate methods to calibrate the DAS channels depth [14].

Tube wave travels along the axis of the borehole in the liquid saturated well with a linear moveout. For each stage in hydraulic fracturing, the tube waves generated from the perforation shots are used to identify the plug position. The plug can be viewed as a reflection interface which reflects the downward propagating tube waves. After the plug location is known, the locations of DAS channels can be determined by comparing the real plug location and the plug location obtained from tube-wave reflections.

DC level is the average amplitude of each trace, which is considered to be zero in ideal situations using Vibroseis truck, namely when there is no bias exists. However, with the temperature fluctuation in the borehole, DC level after simulation is negative above the lowest perforation location and jumps to zero below the lowest perforation location. By analyzing the inflection point of DC level curve, the plug location can be determined. Fig. (15) shows the techniques of tube-wave reflections (a) and DC level jump to determine the depths of DAS channels. It is clear that the tube wave is reflected at the receiver \#432, which is assumed to be the location of plug. By this means, the depth of receiver \# 432 can be determined because the depth of plug is already known and the depths of other receivers can also be calibrated with a constant receiver spacing. Similarly, the DC curve jumped from the negative values which correspond to the depths above the plug to zero which correspond to depths below the plug. So the receiver number corresponds to the plug is determined in the inflection point. Therefore, the depth of other receivers can also be estimated and calibrated.

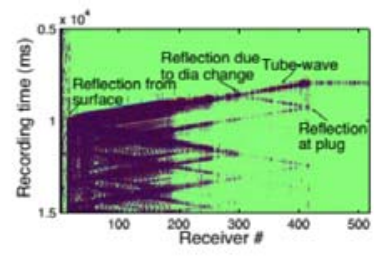

(a)

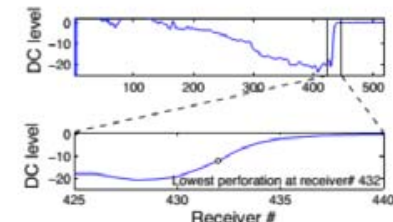

(b)
Fig. (15). (a) common shot gather of original DAS data shows the tube wave reflections that may calibrate the depths (b) DC level jump at the lowest perforation location. The lower figure of $(\mathbf{b})$ is a zoomed part in figure of upper figure [14].

\subsection{Dual Wavefield Component Reconstructed from 1C DAS Data}

Restricted by the limitation of directionality, DAS data are often viewed as 1-C geophone data with the measurements of the strain. However, due to the dense spatial distribution of recording points along the fiber, the particle velocity fields can be obtained by space integration of recorded strain field [23]. The strain field combined with particle velocity field can be used to separate the upward and downward wavefields and the ratio of these two separate fields can provide with the information of local slowness in the formation.

The premise of this construction of particle velocity field is the dense spatial distribution along the fiber, namely the spacing between two adjacent receiver channels should be approximated to $1 \mathrm{~m}$. Then, the vertical particle velocity can be calculated as follows:

$v(z, t)=\frac{\partial}{\partial t} \int_{z} \varepsilon(\eta, t) d \eta$

Where, $v(z, t)$ is the vertical particle velocity; $\varepsilon(\eta, t)$ is the strain field. The integration is performed over the receiver space domain. The local axial slowness at each DAS channel can be calculated as:

$s=\frac{\varepsilon}{v}$

The up-going and down-going field can be separated by following equations:

$D W(z, t)=\frac{\varepsilon(z, t)+F v(z, t)}{2}$
$U P(z, t)=\frac{\varepsilon(z, t)-F v(z, t)}{2}$

Where $D W$ and $U P$ are up-going and down-going field, respectively. $\mathrm{F}$ is the scaling factor determined by formation properties and acquisition parameters. Fig. (16) shows a field example that applies the strain field (a) to obtain the particle velocity field by applying the space integration of the strain field (b). Fig. (17) shows the separated up-going field (c) and down-going field (d) using the strain and particle velocity field according to equation 4 .

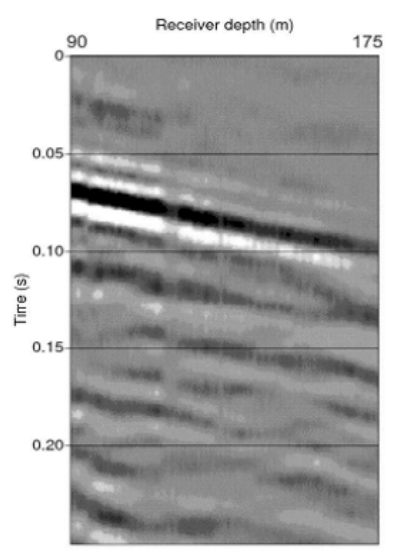

(a)

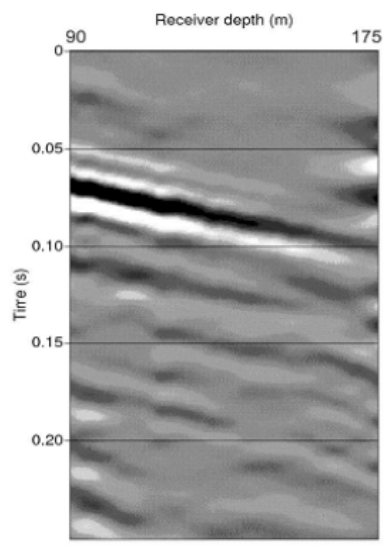

(b)
Fig. (16). The strain field (a) and particle velocity field (b) of DAS VSP data [23].

\subsection{New Design of DAS}

To improve the transverse sensibility of DAS, a helically wrapped cable (HWC) shown in Fig. (18) has been designed [7]. The "cable cut view" here means the depth section view of helically wrapped cable. It shows the projection of helically wrapped cable along the line of " $\mathrm{AB}$ ". The original cable is vertical along the $\mathrm{AB}$ so 
it lacks of the broadside sensitivity while the newly designed cable has a wrapping angle which can improve the sensitivity to the broadside wave. In this design, the wrapping angle $\alpha$ can reach any angle instead of 90 degrees. The response of HWC is determined by the incidence angle $\theta$, the wrapping angle $\alpha$, the properties of the cable and the ground [7]. The HWC prototype with wrapping angle equal to 30 degree has been tested for its broadside sensitivity while the optimal wrapping angle still needs to be tested and investigated. Therefore, the fiber is sensitive to the broadside wave. This new design can be of great help in recording shear waves in near-offset VSP applications.

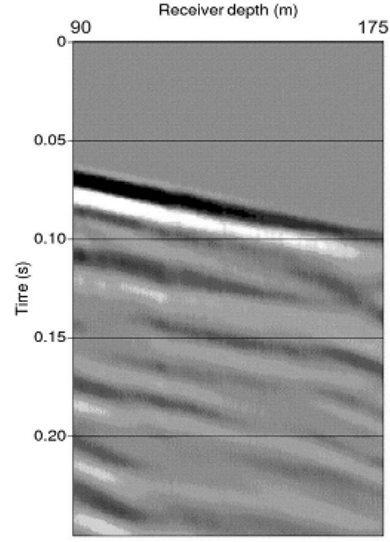

(a)

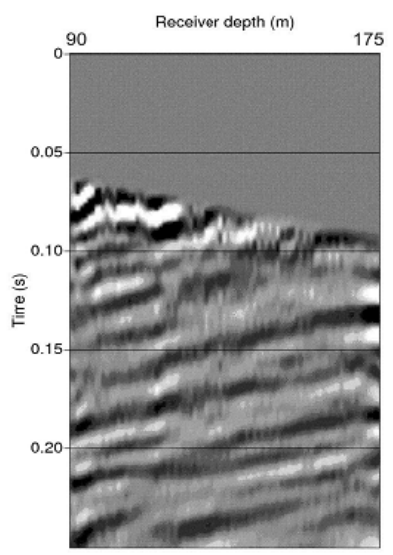

(b)
Fig. (17). The down-going field (a) and up-going field (b) of DAS VSP data [23].
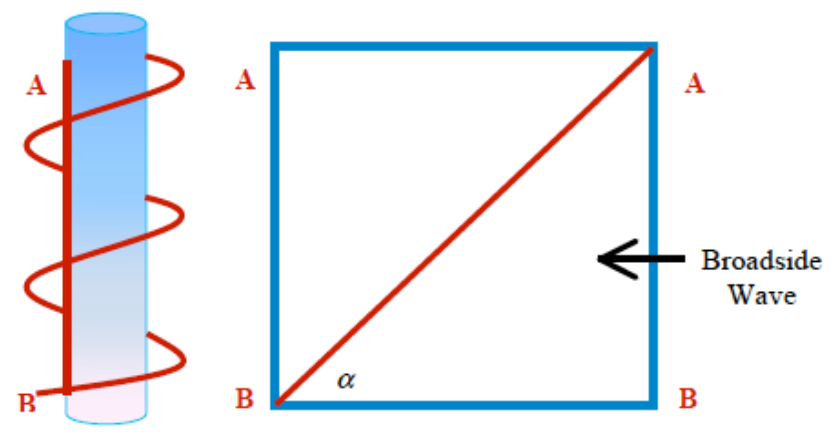

Fig. (18). Helically wrapped cable (left) and its cable cut along line $\mathrm{AB}$ (right) [7].

Fig. (18) shows the structure of HWC and its cable cut view along the axis of the wellbore. Fig. (19) shows a field example that records the seismic measurements with off-line source in horizontal trench for vertical accelerometers, HWC and straight fiber. It's obvious the reflections that propagating perpendicular to cable are absent in straight fiber, while it is clear in HWC, demonstrating the broadside sensitivity of the new designed fiber. Although the HWC is more sensitive to broadside signals, it's still a 1-C measurement. Hence, the direction of incidence of seismic waves remains unknown. To solve the problem, more complex component-selective and broadside-sensitive cables are being designed and tested [7].

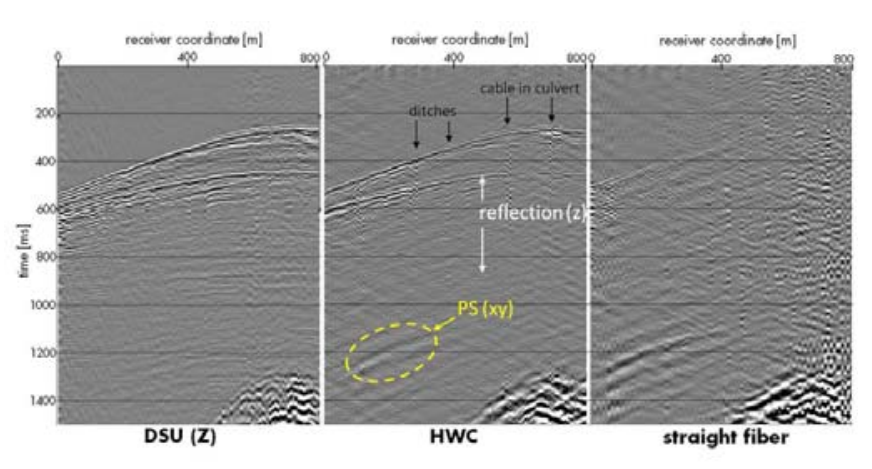

Fig. (19). Field tests of off-line source point in horizontal trench for three different detectors: vertical accelerometers (left), the HWC (middle) and a straight fiber (right) [7].

\section{DISCUSSIONS AND CONCLUSION}

This paper focuses on the review of field data application examples and some pre-processing methods which are applied to alleviate the limitations of Distributed Acoustic Sensing with geophysical measurements in reservoir monitoring, well and reservoir surveillance and microseismic measurements. Although the Distributed Acoustic Sensing technique shows a promising prospect in oil and gas industry, more work should be done to improve its performance.

The most important issue is the enhancement of $\mathrm{S} / \mathrm{N}$ ratio. This can be done by reducing the noise floor of Interrogate Unit, applying a stronger acoustic source (e.g., multiple vibrators) or stacking with more sweeps or signals from multi optical fiber. In addition, the amplitude and phase of scattered signal are captured together along the optical fiber, which means that the data of DAS is nearly linear associated with the strain amplitude. This attribute make it possible to enhance the $\mathrm{S} / \mathrm{N}$ ratio by coherent stacking and correlation. Moreover, besides the band pass filter and median filter mentioned before, wavelet and curvelet transform, with strong ability to analyze the non-stationary signals, may be applied to remove the random noise and extract the expected signals in DAS measurements.

The accurate determination of receiver channels of DAS in depth is another important issue. The technique using tube wave reflections and DC bias is only suitable for downhole applications with plugs (e.g., VSP in hydraulic fracturing monitoring). The application of checkshots may be a more general method to calibrate the receiver channels in depth.

In addition, the DAS measurements are redundant, therefore, a rapid data processing method and visualization for massive data is critical. In addition, the technique of data compression and sparse matrix conversion may be applied prior to the data processing. Moreover, multi-functional optical fiber sensors such as Distributed Temperature Sensing (DTS), Distributed Pressure Sensing (DPS) and Distributed Acoustic Sensing are often installed together in downhole applications. In such situations, comprehensive interpretation with these measurements may substantially lower the ambiguity due to the limitations inherent in a single measurement. 


\section{CONFLICT OF INTEREST}

The authors confirm that this article content has no conflict of interest.

\section{ACKNOWLEDGEMENTS}

This study is supported by the NSFC (Projects NO. 41174118 and NO. 41404100) and one of the major state S\&T special projects (NO. 2011ZX05020). The first author, Meng Li, would like to thank the scholarship support from Shell.

\section{REFERENCES}

[1] J. Mestayer, B. Cox, P. Wills, D. Kiyashchenko, J. Lopez, and M. Costello, "Field trials of distributed acoustic sensing for geophysical monitoring", In: $81^{s t}$ SEG Annual International Meeting, Expanded, San Antonio, USA, 2011, pp. 4253-4257.

[2] D. Miller, T. Parker, S. Kashikar, M. Todorov, and T. Bostick, "Vertical seismic profiling using a fibre-optic cable as a distributed acoustic sensor", In: $74^{\text {th }}$ EAGE Conference and Exhibition, Expanded Abstracts, Copenhagen, Denmark, 2012.

[3] A. Mateeva, J. Mestayer, B. Cox, D. Kiyashchenko, P. Wills, and J. Lopez, "Advances in Distributed Acoustic Sensing (DAS) for VSP”, In: $82^{\text {nd }}$ SEG Annual International Meeting, Expanded Abstracts, Las Vegas, USA, 2012.

[4] A. Mateeva, J. Mestayer, B. Cox, D. Kiyashchenko, P. Wills, and S. Grandi, "Distributed Acoustic Sensing (DAS) for reservoir monitoring with VSP", In: $2^{\text {nd }}$ EAGE Borehole Geophysics Workshop - 3D VSP: Benefits, Challenges and Potential, St. Julain's, Malta, 2013(a).

[5] A. Mateeva, J. Mestayer, Z. Yang, J. Lopez, P. Wills, and J. Roy, "Dual-well 3D VSP in deepwater made possible by DAS", In: 83 $3^{\text {rd }}$ SEG Annual International Meeting, Houston, USA, 2013(b).

[6] A. Mateeva, J. Lopez, J. Mestayer, P. Wills, B. Cox, and D. Kiyashchenko, "Distributed acoustic sensing for reservoir monitoring with VSP", The Leading Edge, vol. 32, no. 10, pp. 1278-1283, 2013(c).

[7] A. Mateeva, J. Lopez, and H. Potters, "Distributed acoustic sensing for reservoir monitoring with vertical seismic profiling”, Geophysical Prospecting, vol. 62, pp. 679-692, 2014.

[8] P. Webster, J. Wall, C. Perkins, and M. Molenaar, "MicroSeismic detection using distributed acoustic sensing", In: $83^{\text {rd }}$ SEG Annual International Meeting, Houston, USA, 2013.

[9] S. G. Karam, P. Webster, K. Hornman, P.G.E. Lumens, A. Franzen, F. Kindy, M. Chiali, and S. Busaidi, "Microseismic Applications using DAS", In: $4^{\text {th }}$ EAGE - Passive Seismic workshop, Optimizing Development of Unconventional Reservoirs, Amsterdam, Netherlands, 2013.

[10] H.J. Der, H.D. Boer, P. Panhuis, R. Kusters, D. Roy, and A. Ridge, "Fibre optic sensing for improved wellbore surveillance", In: $6^{\text {th }}$ IPTC Conference, Beijing, China, 2013.
[11] M. Molenaar, D. Hill, P. Webster, E. Fidan, and B. Birch, "First downhole application of distributed acoustic sensing for hydraulic-fracturing monitoring and diagnostics", In: SPE Drilling and Completion vol. 27, no. 1, pp. 32-38, 2011.

[12] M. Molenaar, and E. Fidan, "Real-time downhole monitoring of hydraulic fracturing treatments using fibre optic distributed temperature and acoustic sensing", In: SPE Unconventional Resources Conference and Exhibition, Vienna, Austria, 2012.

[13] M. Molenaar, "Field cases of hydraulic fracture stimulation diagnostics using fiber optic distributed acoustic sensing (DAS) measurements and Analyses", In: SPE Middle East Unconventional Gas Conference and Exhibition, Muscat, Oman, 2013.

[14] K. Sudhish, P. Wills, and M. Fehler, "Monitoring hydraulic fracturing using Distributed Acoustic Sensing in a treatment well", In: $84^{\text {th }}$ SEG Annual International Meeting, Denver, USA, 2014.

[15] J. Mestayer, S. Grandi, B. Cox, P. Wills, A. Mateeva, and J. Lopez, "Distributed acoustic sensing for geophysical monitoring", In: $74^{\text {th }}$ EAGE Conference and Exhibition, Copenhagen, Denmark, 2012.

[16] T. Parker, S.V. Shatalin, M. Farhadiroushan, Y.I. Kamil, A Gillies, and D. Finfer, "Distributed acoustic sensing - a new tool for seismic applications", In: $74^{\text {th }}$ EAGE Conference and Exhibition, Copenhagen, Denmark, 2012.

[17] T. Parker, S.V. Shatalin, M. Farhadiroushan and D. Miller, "Distributed acoustic sensing: recent field data and performance validation", In: $2^{\text {nd }}$ EAGE Workshop on Permanent Reservoir Monitoring - Current and Future Trends, Extended Abstracts, Stavanger, Norway, 2013.

[18] K. Madsen, S. Dummong, A. Kritski, A. Pedersen, D. Finfer, and A. Gillies, "Simultaneous multiwell VSP in the north Sea using distributed acoustic sensing", In: $75^{\text {th }}$ EAGE Conference \& Exhibition, London, UK, 2013.

[19] T. M. Daley, B. M. Freifeld, J. Franklin, S. Dou, R. Pevzner, V. Shulakova, S. Kashikar, D. E. Miller, J. Goetz, J. Henninges, and S. Lueth, "Field testing of fiber-optic distributed acoustic sensing (DAS) for subsurface seismic monitoring", The Leading Edge, vol. 32, no. 6, pp. 699-706, 2013.

[20] C. Barberan, C. Allanic, D. Avila, J. Billiot, A. Hartog, B. Frignet, and G. Lees, "Multi-offset seismic acquisition using optical fiber behind tubing", In: $74^{\text {th }}$ EAGE Conference \& Exhibition, Copenhagen, Denmark, 2012.

[21] D. Barfoot, "Efficient vertical seismic profiling using fiberoptic distributed acoustic sensing and real-time processing", In: EAGE Borehole Geophysics Workshop II, St. Julain's, Malta, 2013.

[22] J. D. Cocker, E. F. Herkenhoff, M. E. Craven, T. Nemeth, T. M. Daley, D. White, and A. Strudley, "Simultaneous acquisition of das and conventional down-hole geophone array at aqusitore", In: $76^{\text {th }}$ EAGE Conference \& Exhibition, Amsterdam, Netherlands, 2014.

[23] F. Poletto, D. Finfer, P. Corubolo, and B. Farina, "Dual seismic fields from distributed acoustic sensors", In: $84^{\text {th }}$ SEG Annual International Meeting, Denver, USA, 2014.

(C) Li et al.; Licensee Bentham Open.

This is an open access article licensed under the terms of the Creative Commons Attribution Non-Commercial License (http://creativecommons.org/licenses/by-nc/3.0/) which permits unrestricted, non-commercial use, distribution and reproduction in any medium, provided the work is properly cited. 